\title{
Strength-based topology optimisation of plastic isotropic von Mises materials
}

\author{
Herfelt, Morten Andersen; Poulsen, Peter Noe; Hoang, Linh Cao
}

Published in:

Structural and Multidisciplinary Optimization

Link to article, DOI:

$10.1007 / \mathrm{s} 00158-018-2108-y$

Publication date:

2019

Document Version

Peer reviewed version

Link back to DTU Orbit

Citation (APA):

Herfelt, M. A., Poulsen, P. N., \& Hoang, L. C. (2019). Strength-based topology optimisation of plastic isotropic von Mises materials. Structural and Multidisciplinary Optimization, 59(3), 893-906.

https://doi.org/10.1007/s00158-018-2108-y

\section{General rights}

Copyright and moral rights for the publications made accessible in the public portal are retained by the authors and/or other copyright owners and it is a condition of accessing publications that users recognise and abide by the legal requirements associated with these rights.

- Users may download and print one copy of any publication from the public portal for the purpose of private study or research.

- You may not further distribute the material or use it for any profit-making activity or commercial gain

- You may freely distribute the URL identifying the publication in the public portal

If you believe that this document breaches copyright please contact us providing details, and we will remove access to the work immediately and investigate your claim. 


\title{
Strength-based topology optimisation of plastic isotropic von Mises materials
}

\author{
Morten A. Herfelt - Peter N. Poulsen . \\ Linh C. Hoang
}

Received: date / Accepted: date

\begin{abstract}
Conventionally, topology optimisation is formulated as a non-linear optimisation problem, where the material is distributed in a manner which maximises the stiffness of the structure. Due to the nature of non-linear, nonconvex optimisation problems, a multitude of local optima will exist and the solution will depend on the starting point. Moreover, while stress is an essential consideration in topology optimisation, accounting for the stress locally requires a large number of constraints to be considered in the optimisation problem, therefore, global methods are often deployed to alleviate this with less control of the stress field as a consequence. In the present work, a strengthbased formulation with stress-based elements is introduced for plastic isotropic von Mises materials. The formulation results in a convex optimisation problem which ensures that any local optimum is the global optimum, and the problems can be solved efficiently using interior point methods. Four plane stress elements are introduced and several examples illustrate the strength of the convex stress-based formulation including mesh-independence, rapid convergence and near-linear time-complexity.
\end{abstract}

Keywords Topology optimisation · Stress constraints · Plasticity · Von Mises criterion · Second-order cone programming

\section{Introduction}

In the broad sense, topology optimisation is the process of distributing material within a design domain in an optimal manner. The concepts of structural optimisation were discussed as early as in the 19th century and at the turn of the 20th century (see e.g. [27]). The field of structural topology optimisation can be divided into strength optimisation, i.e. limit analysis, and stiffness

Department of Civil Engineering, Technical University of Denmark, Brovej, 2800 Kgs. Lyngby

E-mail: moanh@byg.dtu.dk 
optimisation. Strength optimisation and the concept of fully stressed design received considerable attention in the 1960, 70s, and 80s. Dorn et. al [11] dealt with optimisation of trusses using an equilibrium formulation and linear programming by optimising the size, i.e. the area, of each bar. The equilibrium formulation is similar to that which would later become finite element limit analysis. Prager and Taylor [37] treated the topic of optimal design of both elastic and perfectly plastic structures, while Prager and Shield [36] introduced a general method for optimal plastic design. Michell's work on optimal trusses was extended by Hegemier and Prager [16], and the concept was further expanded to grillages [34,35] as well as archgrids [38]. Kohn and Strang [20] deal with the issue of discrete densities by relaxing the problem and using composite materials. Shape optimisation and the inherited complexities of updating the shape of domains and meshes of finite element models were also treated extensively in the 1980 s (see e.g. $[31,15,39]$ ).

Bendsøe and Kikuchi [4] were some of the first to present the concept of layout optimisation, where the material distribution within a given domain was optimised rather than the shape of the domain. Since then the field has been subject to an extensive research effort. Topology optimisation has since been extended from compliance design to many fields of engineering $[3,10]$. The majority of work within topology optimisation is, nevertheless, concerned with the classic approach of compliance design, where the strain energy is minimised to obtain stiff structures. This approach typically assumes a linear elastic material behaviour and has been extended to several applications within structural engineering, e.g. mechanism design, dynamics, and large displacements (see References $[5,10]$ for extensive literature reviews).

Density-based approaches are some of the most widely used methodologies in conventional topology optimisation. One of the strengths of conventional stiffness-based topology optimisation is the solution scheme, where only few constraints are included in the optimisation routine and the finite element equations are solved outside the optimisation routine. Continuous density variables are desirable due to the challenges of solving large scale integer optimisation problems and are therefore commonly used. Nevertheless, a black-white design is often desirable and penalty methods are used to force the solutions towards black-white designs, e.g the popular Solid Isotropic Material with Penilisation (SIMP) method [41]. Conventional topology optimisation is prone to numerical issues such as mesh-dependency and checkerboarding [41], however, various filter methods are commonly deployed to alleviate this resulting in a method with several parameters influencing the optimal solution. The conventional formulation of density-based topology optimisation including penalisation and filters leads to a non-linear, non-convex optimisation problem. These problems have a multitude of local optima and the solution will therefore be dependant on the starting point as well as the solution scheme, e.g. MMA [44] and the optimality criterion algorithm [40] may find different solutions.

It is essential to consider the stress field in the design of structures, but the number of publications considering stress constraints is rather limited [13]. This is primarily due to the singularity phenomenon and the local nature of 
stress constraints [5]. The various techniques to constrain the stress field can be classified as either local methods, global methods, or regional methods. Local methods impose stress constraints on the element level, however, this leads to a large number of constraints in the optimisation problem. Local constraints are nevertheless needed when considering materials which e.g. behave differently in tension and compression [9]. Global methods, on the other hand, deploy some measure to combine the local stress states into a single criterion, e.g. a $p$-norm measure [12].

In this work, a finite element approach with stress-based elements is used to obtain a convex optimisation problem based on the work equation and local stress constraints, i.e. the yield function enforced locally. A convex formulation ensures that any local optimum will be the global optimum, and the solution will therefore not depend on the starting point. Moreover, the proposed formulation has virtually no mesh dependencies and is not prone to checkerboarding, hence, filters and similar are not needed. The formulation is similar to finite element limit analysis (FELA) $[1,33,17]$ and the set of feasible stress states are governed by the yield function assuming a rigid plastic behaviour. Kammoun and Smaoui [18] presented a formulation for topology optimisation based on FELA for plane strain Tresca materials. A lower bound approach was taken, which ensures a statically admissible stress field, whereas in this paper, we consider four different elements including the upper bound element, which ensures a kinematically feasible solution. Identically to [18], the scope is to minimise the volume of the structure for a given load case while the stress field satisfies the yield function in the chosen stress interpolation points. In many ways, the present formulation is identical to sizing optimisation, where e.g. the cross sectional area of a bar is varied to minimise some cost function, and in this sense it can be seen as a direct continuation where the variables, i.e. the densities, can be interpreted as the thickness of the structure.

For FELA, the solution is governed by the strength rather than the stiffness, and in this paper an isotropic von Mises material is assumed. Pedersen [32] showed that the optimal strength design and the optimal compliance design will be identical if the yield criterion is consistent with the elastic energy measure. For the von Mises criterion, this corresponds to an incompressible material, i.e. with a Poisson's ratio of $\nu=0.5$. The von Mises criterion can be formulated exact in a closed form using conic constraints, and the resulting optimisation problem will be a second-order cone program (SOCP), a subclass of convex optimisation, which can be solved extremely efficiently by use of interior point methods. Finally, four examples are used to show the strengths and advantages of the presented framework. 


\section{Formulation and finite elements}

2.1 Volume minimisation

The formulation of strength-based topology optimisation is based on the work equation similar to finite element limit analysis (FELA). Identically to FELA, the resulting problem is a convex optimisation problem. As mentioned in the introduction, we use a density-based approach, hence, a normalised density field $\rho$ is introduced. The yield strength of the material $f_{y}$ is assumed to be proportional to the density,

$$
f_{y}=\rho f_{y}^{0}
$$

where $f_{y}^{0}$ is the maximum yield strength, and the density field can only take values between 0 and 1, i.e. $\rho \in[0,1]$. We now consider a solid body occupying a volume $V$. The body is supported on the boundary $S_{u}$ and subject to external loading $\boldsymbol{t}$ on the boundary $S_{\sigma}$ as well as the body forces $\boldsymbol{b}$ acting on the volume, see Fig. 1.

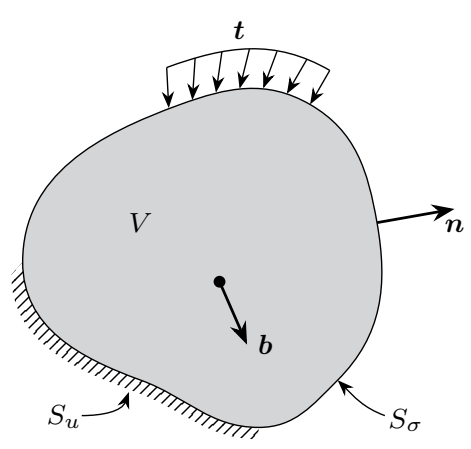

Fig. 1 Body subjected to external forces $\boldsymbol{t}$ and body forces $\boldsymbol{b}$.

The behaviour of the body under the applied loads are governed by a set of equations. First and foremost, equilibrium for the body forces is ensued by the following equation:

$$
\boldsymbol{\nabla}^{T} \boldsymbol{\sigma}+\boldsymbol{b}=\mathbf{0} \quad \text { in } V
$$

Next, equilibrium at the boundary $S_{\sigma}$ is ensured as follows:

$$
\mathbf{P} \boldsymbol{\sigma}=\boldsymbol{t} \quad \text { on } S_{\sigma}
$$

The supports on the boundary $S_{u}$ gives the following equation:

$$
\boldsymbol{u}=\mathbf{0} \quad \text { on } S_{u}
$$

And finally, we have the strain-displacement relationship:

$$
\varepsilon=\nabla u
$$


Moreover, the stress field must satisfy the yield criterion, which in this case also is a function of the density, $\rho$ :

$$
f(\boldsymbol{\sigma}, \rho) \leq 0 \quad \text { in } V
$$

Minimising the total volume of the structure gives the following optimisation problem where the stress field $\boldsymbol{\sigma}$ and the density field $\rho$ are the variable quantities:

$$
\begin{array}{ll}
\text { minimise } & \int_{V} \rho \mathrm{d} V \\
\text { subject to } & \boldsymbol{\nabla}^{T} \boldsymbol{\sigma}+\boldsymbol{b}=\mathbf{0}, \quad \text { in } V \\
& \mathbf{P} \boldsymbol{\sigma}=\boldsymbol{t}, \quad \text { on } S_{\sigma} \\
& f(\boldsymbol{\sigma}, \rho) \leq 0, \quad \text { in } V
\end{array}
$$

Utilising the standard Lagrange multiplier technique, we transfer the second constraint to the objective function by multiplying by a Lagrange multiplier and integrating the product over the boundary. In this case, the Lagrange multipliers can be interpreted as the displacements and will be denoted $\boldsymbol{u}$ :

$$
\begin{array}{cl}
\underset{\rho}{\min \min _{u}} & \int_{V} \rho \mathrm{d} V+\int_{S_{\sigma}} \boldsymbol{u}^{T}(\mathbf{P} \boldsymbol{\sigma}-\boldsymbol{t}) \mathrm{d} S_{\sigma} \\
\text { subject to } & \boldsymbol{\nabla}^{T} \boldsymbol{\sigma}+\boldsymbol{b}=\mathbf{0}, \quad \text { in } V \\
& f(\boldsymbol{\sigma}, \rho) \leq 0, \quad \text { in } V
\end{array}
$$

Using the conventional finite element methodology, we divide the domain into finite elements. For each element, the stresses and displacements are approximated using shape functions:

$$
\begin{aligned}
& \boldsymbol{\sigma} \approx \mathbf{N}_{\sigma} \hat{\boldsymbol{\sigma}} \\
& \boldsymbol{u} \approx \mathbf{N}_{u} \hat{\boldsymbol{u}}
\end{aligned}
$$

where $\hat{\boldsymbol{\sigma}}$ and $\hat{\boldsymbol{u}}$ are the discretised stresses and displacements, respectively, and $\mathbf{N}_{\sigma}$ and $\mathbf{N}_{u}$ are the associated shape functions. Moreover, the density field is likewise discretised:

$$
\rho \approx \mathbf{N}_{\rho} \hat{\rho}
$$

We now consider a single element and substitutes the approximations into (8):

$$
\begin{array}{cl}
\min _{\rho} \min _{u} & \boldsymbol{v}^{T} \hat{\rho}+\hat{\boldsymbol{u}}^{T} \hat{\mathbf{B}}^{T} \hat{\boldsymbol{\sigma}}-\hat{\boldsymbol{u}}^{T} \hat{\boldsymbol{t}} \\
\text { subject to } & \mathbf{S}^{T} \hat{\boldsymbol{\sigma}}+\hat{\boldsymbol{b}}=\mathbf{0} \\
& f(\hat{\boldsymbol{\sigma}}, \hat{\rho}) \leq 0
\end{array}
$$

with

$$
\begin{aligned}
& \hat{\mathbf{B}}^{T}=\int_{S} \mathbf{N}_{u}^{T} \mathbf{P} \mathbf{N}_{\sigma} \mathrm{d} S \\
& \hat{\boldsymbol{t}}=\int_{S} \mathbf{N}_{u}^{T} \boldsymbol{t} \mathrm{d} S \\
& \boldsymbol{v}=\int_{V} \mathbf{N}_{\rho} \mathrm{d} V
\end{aligned}
$$


By solving the displacement part of (11) first and incorporating the optimality condition as a constraint, we then obtain the final minimisation problem:

$$
\begin{array}{cl}
\min _{\rho} & \boldsymbol{v}^{T} \hat{\rho} \\
\text { subject to } & \hat{\mathbf{B}}^{T} \hat{\boldsymbol{\sigma}}=\hat{\boldsymbol{t}} \\
& \mathbf{S}^{T} \hat{\boldsymbol{\sigma}}+\hat{\boldsymbol{b}}=\mathbf{0} \\
& f(\hat{\boldsymbol{\sigma}}, \hat{\rho}) \leq 0
\end{array}
$$

The matrix $\mathbf{S}^{T}$ depends on the individual element and will be given in Sec. 2.4 .

\subsection{Yield function}

In this paper we consider the von Mises criterion for plane stress which is based on the second stress invariant, $J_{2}$, and has the compact form

$$
\sqrt{3 J_{2}} \leq f_{y}=f_{y}^{0} \rho_{i}
$$

where $J_{2}$ is given as

$$
J_{2}=\frac{1}{6}\left(\sigma_{x}-\sigma_{y}\right)^{2}+\frac{1}{6}\left(\sigma_{y}-\sigma_{z}\right)^{2}+\frac{1}{6}\left(\sigma_{z}-\sigma_{x}\right)^{2}+\tau_{x y}^{2}+\tau_{y z}^{2}+\tau_{z x}^{2}
$$

In the two-dimensional case, $J_{2}(15)$ is reduced to

$$
J_{2}=\frac{1}{6}\left(\sigma_{x}-\sigma_{y}\right)^{2}+\frac{1}{6} \sigma_{y}^{2}+\frac{1}{6} \sigma_{x}^{2}+\tau_{x y}^{2}
$$

By introducing three auxiliary variables,

$$
\alpha_{1}=\sigma_{x}-\frac{1}{2} \sigma_{y}, \quad \alpha_{2}=\frac{\sqrt{3}}{2} \sigma_{y}, \quad \alpha_{3}=\sqrt{3} \tau_{x y},
$$

the von Mises criterion can be rewritten as

$$
f_{y}^{0} \rho \geq \sqrt{\alpha_{1}^{2}+\alpha_{2}^{2}+\alpha_{3}^{2}},
$$

or

$$
f_{y}^{0} \hat{\rho} \geq \sqrt{\hat{\boldsymbol{\alpha}}^{T} \hat{\boldsymbol{\alpha}}}
$$

which has the shape of a quadratic cone $[6,22]$. For the von Mises criterion, the formulation (13) can be expanded to obtain the following second-order cone program:

$$
\begin{array}{lll}
\operatorname{minimise} & \boldsymbol{v}^{T} \hat{\boldsymbol{\rho}} & \\
\text { subject to } & \hat{\mathbf{B}}^{T} \hat{\boldsymbol{\sigma}}=\hat{\boldsymbol{t}}, & \\
& \mathbf{S}^{T} \hat{\boldsymbol{\sigma}}+\hat{\boldsymbol{b}}=\mathbf{0}, & \\
& f_{y}^{0} \hat{\rho}_{i} \geq \sqrt{\left(\mathbf{C} \hat{\boldsymbol{\sigma}}_{i}\right)^{T}\left(\mathbf{C} \hat{\boldsymbol{\sigma}}_{i}\right)}, & i=1,2, \ldots, m \\
& \hat{\rho}_{i} \in[0,1], & i=1,2, \ldots, m
\end{array}
$$


where $i$ refers to the $i$ th stress node and the associated stresses, $\boldsymbol{\sigma}_{i}$ and density $\rho_{i}$. The matrix $\mathbf{C}$ can be given as:

$$
\mathbf{C}=\left[\begin{array}{ccc}
1 & -\frac{1}{2} & 0 \\
0 & \frac{\sqrt{3}}{2} & 0 \\
0 & 0 & \sqrt{3}
\end{array}\right]
$$

Large-scale optimisation problems (20) will be banded and display extreme sparsity, i.e. low number of non-zeros to zeros. This is exploited by state-ofthe-art solvers and the problems can be solved remarkably efficient, which will be shown in the following sections.

\subsection{Second-order cone programming}

Second-order cone programming (SOCP) is a class of convex optimisation problems, which is a generalisation of linear programming and a subclass of semidefinite programming. SOCP is used widely in many fields of engineering and science today $[23,8]$.

SOCP features a linear objective function which is minimised over the intersection of an affine set and the Cartesian product of second-order cones [2]. First and foremost, we introduce the set of the $k$-dimensional second-order cone:

$$
\mathcal{Q}_{k}=\left\{\boldsymbol{x} \mid \boldsymbol{x} \in \mathbb{R}^{k}, x_{1} \geq\left\|x_{2: k}\right\|_{2}\right\}
$$

The notation $\boldsymbol{x} \in \mathcal{Q}_{k}$ is therefore equal to

$$
\boldsymbol{x} \in \mathcal{Q}_{k} \quad \Leftrightarrow \quad x_{1} \geq\left\|\boldsymbol{x}_{2: k}\right\|_{2}=\sqrt{\boldsymbol{x}^{T} \boldsymbol{x}}=\sqrt{x_{2}^{2}+x_{3}^{2}+\cdots+x_{k}^{2}}
$$

It is seen that (23) has the same shape as the von Mises criterion (19). We now define $\mathcal{Q}$ as the Cartesian product of second-order cones,

$$
\mathcal{Q}=\mathcal{Q}_{k_{1}} \times \mathcal{Q}_{k_{2}} \times \cdots \times \mathcal{Q}_{k_{q}},
$$

hence,

$$
\boldsymbol{x} \in \mathcal{Q} \quad \Leftrightarrow \quad x_{1} \in \mathcal{Q}_{k_{1}}, \quad \boldsymbol{x}_{2} \in \mathcal{Q}_{k_{2}}, \quad \ldots, \quad \boldsymbol{x}_{q} \in \mathcal{Q}_{k_{q}},
$$

where $\boldsymbol{x}_{i}$ are parts of $\boldsymbol{x}$ associated with the $i$ th cone. The standard form of SOCP is commonly stated as:

$$
\begin{array}{ll}
\text { minimise } & \boldsymbol{g}^{T} \boldsymbol{x} \\
\text { subject to } & \mathbf{A} \boldsymbol{x}=\boldsymbol{b} \\
& \boldsymbol{x} \in \mathcal{Q}
\end{array}
$$

where the vector $\boldsymbol{x}$ contains the problem variables. The matrix $\mathbf{A}$ and the vector $\boldsymbol{b}$ define the linear equality constraints, while the vector $\boldsymbol{g}$ defines the linear objective function. 
The problem (26) is commonly denoted the primal problem, which has the following dual problem:

$$
\begin{array}{ll}
\operatorname{maximise} & \boldsymbol{b}^{T} \boldsymbol{y} \\
\text { subject to } & \mathbf{A}^{T} \boldsymbol{y}+\boldsymbol{s}=\boldsymbol{g} \\
& \boldsymbol{s} \in \mathcal{Q}
\end{array}
$$

where $\boldsymbol{y}$ and $\boldsymbol{s}$ are the variables of the dual problem. The primal and dual problems are solved simultaneously, and the dual problem (27) provides additional information about the primal problem. For this work, the dual problem will contain information on the strain velocities and displacement velocities.

Second-order cone programming can be solved remarkably efficient using interior point methods, a class of polynomial time algorithms originally proposed by Kamarkar [19]. Modern solvers are capable of solving problems with hundreds of thousands of variables in a matter of minutes on laptop computers. For an introduction to state-of-the-art solvers in convex optimisation, the reader is referred to References $[2,45]$.

\subsection{Plane stress elements}

Four plane stress elements are considered in the present framework. All finite elements feature a linear stress field described by three stress nodes and a quadratic displacement field described by six displacement nodes, see Fig. 2.

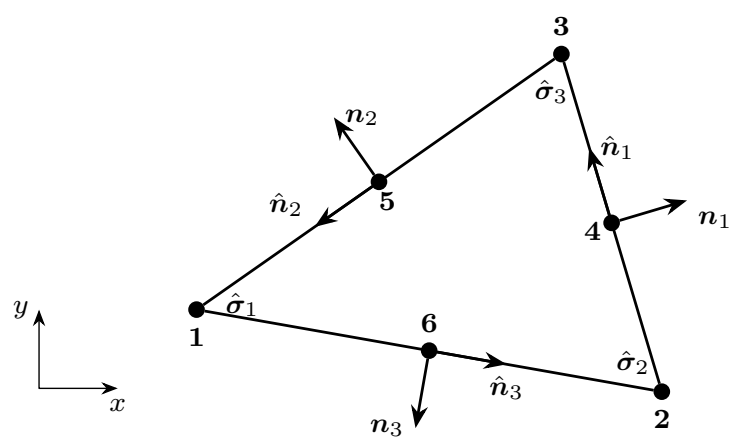

Fig. 2 Geometry of the triangular, six-node elements: Unit normal vectors to the three sides and stress vectors are shown.

The linear stress field is given by three stress vectors, one for each corner. These stress vectors are collected in an element stress vector, $\hat{\boldsymbol{\sigma}}_{e l}$ :

$$
\hat{\boldsymbol{\sigma}}_{e l}=\left[\begin{array}{c}
\hat{\boldsymbol{\sigma}}_{1} \\
\hat{\boldsymbol{\sigma}}_{2} \\
\hat{\boldsymbol{\sigma}}_{3}
\end{array}\right], \quad \text { with } \quad \hat{\boldsymbol{\sigma}}_{i}=\left[\begin{array}{c}
\sigma_{x i} \\
\sigma_{y i} \\
\tau_{x y i}
\end{array}\right]
$$


Associated with each side $i$, we have a unit normal vector:

$$
\boldsymbol{n}_{i}=\left[\begin{array}{c}
n_{x}^{i} \\
n_{y}^{i}
\end{array}\right]
$$

Moreover, we define the following stress-to-traction arrays:

$$
\begin{aligned}
& \mathbf{P}_{i}=\left[\begin{array}{ccc}
n_{x}^{i} & 0 & n_{y}^{i} \\
0 & n_{y}^{i} & n_{x}^{i}
\end{array}\right] \\
& \tilde{\mathbf{P}}_{i}=l_{i} \mathbf{P}_{i}
\end{aligned}
$$

where $l_{i}$ is the length of side $i$.

\subsubsection{Relaxed lower bound element}

Lower bound elements ensure a statically admissible stress field, i.e. traction continuity in the case of $2 \mathrm{D}$ problems. The formulation of the lower bound element is given by Sloan [42], however, the element has some issues in regards to linear dependencies [25].

A relaxed version of the lower bound element, i.e. a mixed element, will be used in this paper instead. The element was originally formulated by Krabbenhoft [21]. Due to the linear stress field, the tractions vary linearly as well along the element sides and can be stated as

$$
\boldsymbol{t}_{i}(s)=\left(\frac{1}{2}-s\right) \mathbf{P}_{i} \hat{\boldsymbol{\sigma}}_{k}+\left(s-\frac{1}{2}\right) \mathbf{P}_{i} \hat{\boldsymbol{\sigma}}_{j}
$$

where $j$ and $k$ refer to the stress nodes (see Fig. 2), $i$ refers to the side number (opposite node $i$ ), and $i, j$, and $k$ are permutations of 1,2 , and 3 . Moreover, $s \in$ $[-1 / 2,1 / 2]$ is a dimensionless length coordinate. To obtain a relaxed version of the lower bound element, the tractions are distributed to the nodes according to the quadratic displacement field described by the shape functions $N_{j}^{i}(s)$ :

$$
\boldsymbol{q}_{j}^{i}=l_{i} \int_{-1 / 2}^{1 / 2} N_{j}^{i}(s) \boldsymbol{t}_{i}(s) \mathrm{d} s
$$

where $\boldsymbol{q}_{j}^{i}$ is the contribution to the nodal force in node $j$ from the tractions along side $i$. The equilibrium matrix for the relaxed lower bound element can now be stated as follows:

$$
\hat{\mathbf{B}}^{T}=-\frac{1}{6}\left[\begin{array}{ccc}
\tilde{\mathbf{P}}_{1} & 0 & 0 \\
0 & \tilde{\mathbf{P}}_{2} & 0 \\
0 & 0 & \tilde{\mathbf{P}}_{3} \\
0 & 2 \tilde{\mathbf{P}}_{2}+2 \tilde{\mathbf{P}}_{3} 2 \tilde{\mathbf{P}}_{3}+2 \tilde{\mathbf{P}}_{2} \\
2 \tilde{\mathbf{P}}_{1}+2 \tilde{\mathbf{P}}_{3} & 0 & 2 \tilde{\mathbf{P}}_{3}+2 \tilde{\mathbf{P}}_{1} \\
2 \tilde{\mathbf{P}}_{1}+2 \tilde{\mathbf{P}}_{2} 2 \tilde{\mathbf{P}}_{2}+2 \tilde{\mathbf{P}}_{1} & 0
\end{array}\right]
$$


A central displacement node is needed for the original lower bound element, and this is carried over to the relaxed element. The element may be subjected to a uniformly surface load $\boldsymbol{p}$, which give rise to the following differential equation:

$$
\begin{aligned}
& \frac{\partial \sigma_{x}}{\partial x}+\frac{\partial \tau_{x y}}{\partial y}+p_{x}=0 \\
& \frac{\partial \sigma_{y}}{\partial y}+\frac{\partial \tau_{x y}}{\partial x}+p_{y}=0
\end{aligned}
$$

Introducing shape functions for the linear stress field, (34) can be written as

$$
\begin{gathered}
A\left[\begin{array}{c}
p_{x} \\
p_{y}
\end{array}\right]-\frac{1}{2}\left[\begin{array}{lll}
\tilde{\mathbf{P}}_{1} & \tilde{\mathbf{P}}_{2} & \tilde{\mathbf{P}}_{3}
\end{array}\right] \hat{\boldsymbol{\sigma}}_{e l}=0 \\
\mathbf{S}^{T}=-\frac{1}{2}\left[\begin{array}{lll}
\tilde{\mathbf{P}}_{1} & \tilde{\mathbf{P}}_{2} & \tilde{\mathbf{P}}_{3}
\end{array}\right], \quad \hat{\boldsymbol{b}}=A\left[\begin{array}{l}
p_{x} \\
p_{y}
\end{array}\right]
\end{gathered}
$$

The relaxed lower bound element is compared to three plane stress elements, namely two mixed elements, the Zouain element [7] and the standard six-node element [22], as well as the upper bound element [43]. All three elements have been used extensively in the literature, and the equilibrium matrix for the elements can be found in the appendix.

The Zouain element is a mixed element, which uses the corner nodes as stress interpolation points and the midside nodes as integration points. For finite element limit analysis, the element was first proposed by Borges et al. [7]. The element does not bound the exact solution, but it will be more conservative than the upper bound and standard six-node elements.

The standard six-node element uses stress interpolation and integration points which are located at $\left(\lambda_{j-1}, \lambda_{j}, \lambda_{j+1}\right)=\left(\frac{1}{6}, \frac{4}{6}, \frac{1}{6}\right), j=1,2,3$, where $\lambda_{j}$ are triangle area coordinates [22]. The element is widely used in finite element analysis due to the accuracy.

In finite element limit analysis, the upper bound element will ensure a kinematically admissible collapse mode and can be used to bracket the exact solution. The formulation of the upper bound element was given by Sloan [43] and has been used extensively since (see e.g. References $[24,26,22]$ ). The upper bound element will in this work generally give the least conservative design, i.e. the solution which requires the least material.

\subsection{Densities as integers}

The presented formulation assumes that the actual strength, $f_{y}$, is proportional to the density. This approach will necessarily lead to a grey-scale design, i.e. a design with densities in-between zero and one. As discussed in the introduction, in topology optimisation the SIMP approach [41] is often used to push the 
design towards a black-white design by introducing a penalty on grey-scale. This is done by relating the actual stiffness to the density to some power $p$ :

$$
\mathbf{C}_{i j k l}(x) \leq \mathbf{C}_{i j k l}^{0} \rho(x)^{p}, \quad p \geq 1
$$

where $\mathbf{C}_{i j k l}^{0}$ is the stiffness tensor and $p$ is the penalisation factor. A penalisation factor of 3 is usually sufficient to ensure a reasonable black-white design [40]. For penalisation factors larger than 1, the relation (37) is concave and will therefore not fit the presented convex formulation. For two-dimensional problems, the density can be interpreted as the thickness, hence, a grey-scale design can be desirable. This is especially true when considering $3 \mathrm{D}$ printed reinforced concrete structures, where the thickness can be varied to create optimal structures. Moreover, this interpretation is straight forward and does not pose any requirements to the microstructure of the given material. Nevertheless, penalisation or similar schemes can be incorporated in a non-convex generalisation of the formulation, possibly using the convex solution as the starting guess.

Alternatively, treating the densities as integers guarantee a black-white design. The problem is now a so-called mixed-integer second-order cone program (MISOCP) and can be stated as:

$$
\begin{array}{lll}
\text { minimise } & \boldsymbol{v}^{T} \hat{\boldsymbol{\rho}} \\
\text { subject to } & \hat{\mathbf{B}}^{T} \hat{\boldsymbol{\sigma}}=\hat{\boldsymbol{t}}, & \\
& \mathbf{S}^{T} \hat{\boldsymbol{\sigma}}+\hat{\boldsymbol{b}}=\mathbf{0}, & \\
& f_{y}^{0} \hat{\rho}_{i} \geq \sqrt{\left(\mathbf{C} \hat{\boldsymbol{\sigma}}_{i}\right)^{T}\left(\mathbf{C} \hat{\boldsymbol{\sigma}}_{i}\right)}, i=1,2, \ldots, m \\
& \hat{\rho}_{i} \in[0,1], & i=1,2, \ldots, m \\
& \hat{\boldsymbol{\rho}} \in \mathbb{Z}^{m} &
\end{array}
$$

where $\mathbb{Z}$ is the set of integers. The vast majority of variables of the problem (38) will be continuous while few will be integers. Generally, optimisation problems involving integers are difficult and extremely slow to solve. For continuous optimisation problems, e.g. linear programming or second-order cone programming, algorithms with a polynomial worst-case complexity have been developed during the last three decades (see e.g. References [19,30,2]). While integer programming is used in many branches of mathematics and engineering, the problems are classified as NP-hard problems and have an exponential worst-case time complexity [29].

\section{Applications}

Four examples will be presented in this section. Based on the four examples, the convergence rate of the elements and the computational time will be analysed. The mesh dependency and other numerical issues will be discussed as well.

Many of the commonly featured examples in topology optimisation involves point loads and point supports. Using local stress constraints, point loads and 
supports are not feasible as they will give rise to local stress fields which cannot satisfy the yield criterion. Supports and loads are therefore applied over a small area instead.

The meshes presented in this paper is generated using Mesh2D for Matlab [14], and the optimisation problems are solved using the commercial solver MOSEK 8.0 [28]. The presented examples are solved on a laptop with an Intel i7-6500 CPU $(2.50 \mathrm{GHz}, 4$ cores). All calculated volume fractions and computational times are listed in the appendix.

\subsection{MBB-beam}

The MBB-beam is a commonly used example for topology optimisation (see e.g. [40]), and the beam will be examined here as well. The beam is subjected to a (point) load acting in the centre and supported in both ends in the vertical direction.

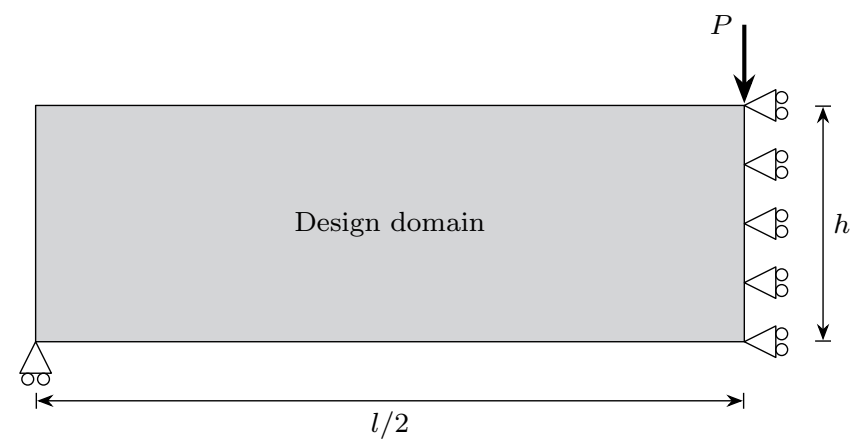

Fig. 3 MBB-beam subjected to a point load. The symmetry is exploited and only half of the beam is modelled.

The domain of the analysed MBB-beam has a total length of $l=36$ and a height of $h=6$. As discussed, point loads and point supports may lead to infeasible local stress fields, hence, infeasible optimisation problems. The load, $P$, seen in Fig. 3 is therefore distributed over a length of $s=0.5$. Similarly, the point support near the leftmost end is modelled as a edge support over a length of $s=0.5$. The load $P$ has a magnitude of 50, while a yield strength of $f_{y}^{0}=100$ is used for the present example.

Fig. 4 shows the optimal design of the MBB-beam for the four elements where black indicates a volume fraction of one and white indicates a volume fraction of zero. The relaxed lower bound element requires the largest volume $(19.75 \%)$ while the upper bound element requires the smallest volume (19.59 $\%$ ), which is to be expected. Nevertheless, all designs shown in Fig. 4 are practically identical featuring thick flanges at midspan, where the bending moment will be largest. In-between the flanges, a grey-scale area indicate that 


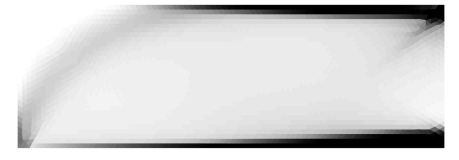

(a) Relaxed lower: $\boldsymbol{V}^{T} \hat{\boldsymbol{\rho}}=0.1975$

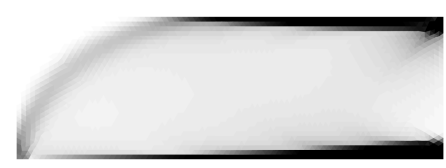

(c) Standard: $\boldsymbol{V}^{T} \hat{\boldsymbol{\rho}}=0.1962$

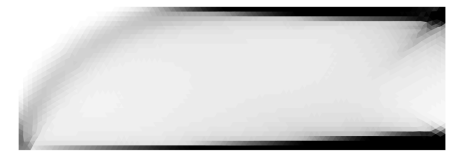

(b) Zouain: $\boldsymbol{V}^{T} \hat{\boldsymbol{\rho}}=0.1969$

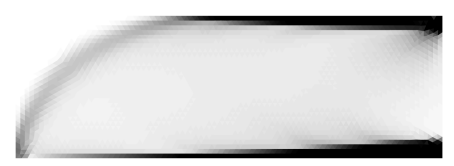

(d) Upper: $\boldsymbol{V}^{T} \hat{\boldsymbol{\rho}}=0.1959$

Fig. 4 Optimal designs of the MBB-beam problem for the four elements using 4,988 elements.

a smaller amount of material is sufficient to carry the shear. Finally, an archlike structure is produced near the supports.

Fig. 5(a) shows that all four elements rapidly converge towards a common volume fraction and design. For the coarsest mesh, the volume fraction of all four elements are larger than the optimal design - even for the upper bound element. At some point, however, the upper bound element reaches a minimum and starts approaching the optimal design from below as expected.

The computational time for solving the optimisation problem is shown in Fig. 5(b) in a double logarithmic coordinate system. The curves for all four elements appear linear, hence, the time-complexity is polynomial. The dashed line represents a curve for $O\left(n^{1.1}\right)$ in order to visualise that the computational time of all four elements is approximately proportional to the problem size to the power of 1.1 .

\subsection{Cantilever beam}

The next example features a deep cantilever beam subjected to a point load. The beam is supported in the left hand side and the design domain is a rectangle with the length $l=32$ and the height $h=20$, see Fig. 6 . The point load is distributed over a length of $s=1$ and has a magnitude of 100 . Like the previous example, the yield strength of $f_{y}^{0}=100$ is used here.

Fig. 7 that shows the solution, i.e. the design, is independent of the mesh. A refined mesh gives a refined solution, but the overall design is unchanged. Conventional topology optimisation, on the other hand, often requires filters to obtain mesh independence. The design features an arch-like structure (similar to the MBB-beam problem). Near the supports on the left hand side, material is almost exclusively placed in the top and bottom in order to carry the bending moment. 


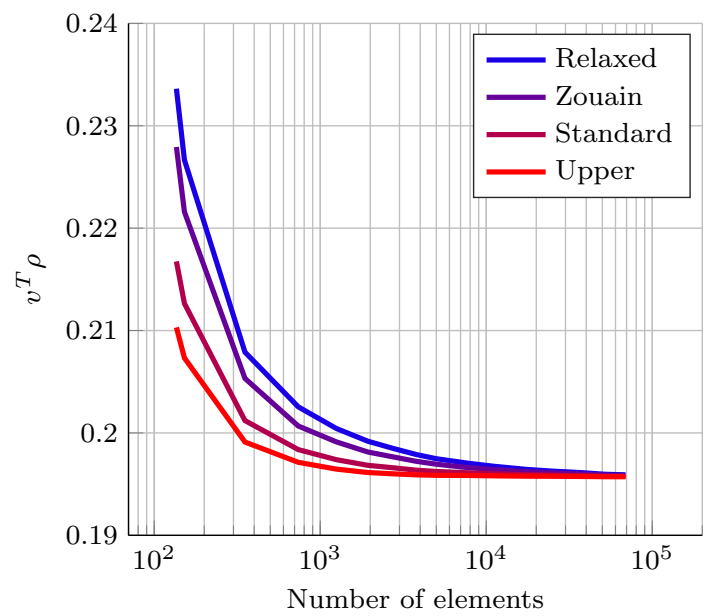

(a)

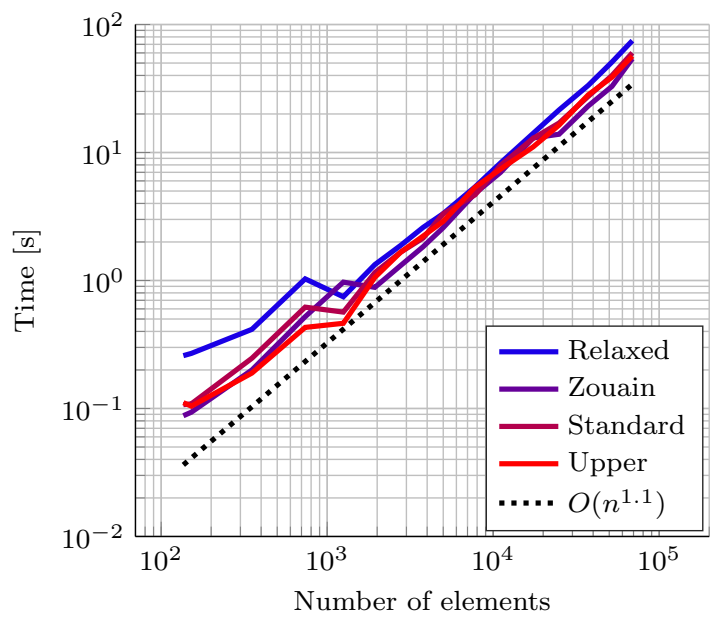

(b)

Fig. 5 MBB-beam problem: a) Convergence for the four elements, b) computational time for the four elements.

The convergence and time-complexity for the cantilever beam problem display the same tendencies as for the MBB-beam problem. Fig. 8(a) shows that all elements rapidly converge towards a common volume fraction. It is noticed, however, that even the upper bound element exclusively approach from above, but it is still the least conservative design, i.e. lowest volume fraction. Fig. 8(b) shows that the computational time is approximately proportional to the problem size to the power of 1.1 - similar to the MBB-beam problem. 


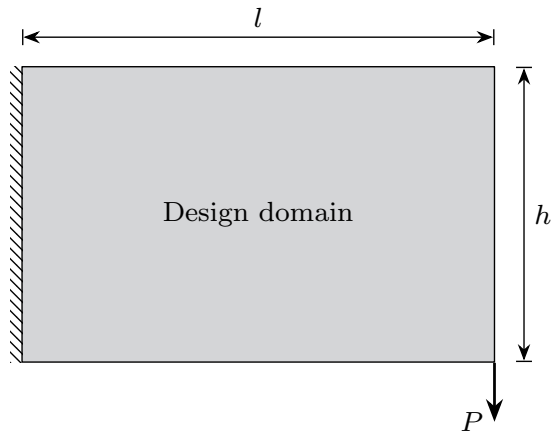

Fig. 6 Deep cantilever beam subjected to a point load at the bottom right corner. Left boundary is assumed fixed.

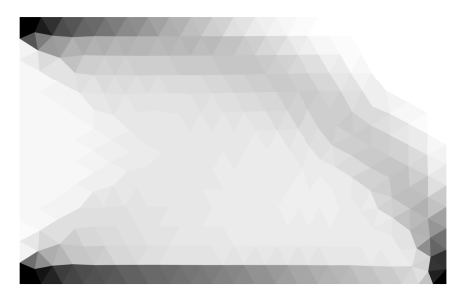

(a) 576 elements, $\boldsymbol{V}^{T} \hat{\boldsymbol{\rho}}=0.1603$

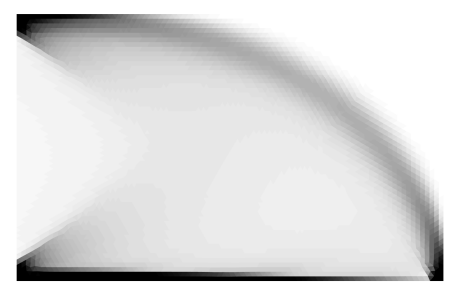

(c) 9,297 elements, $\boldsymbol{V}^{T} \hat{\boldsymbol{\rho}}=0.1582$

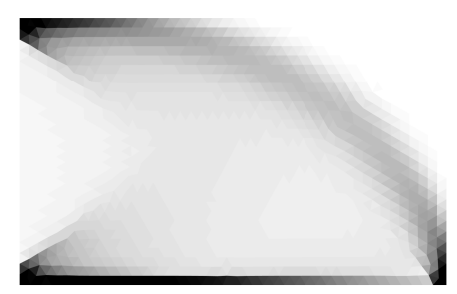

(b) 2,331 elements, $\boldsymbol{V}^{T} \hat{\boldsymbol{\rho}}=0.1587$

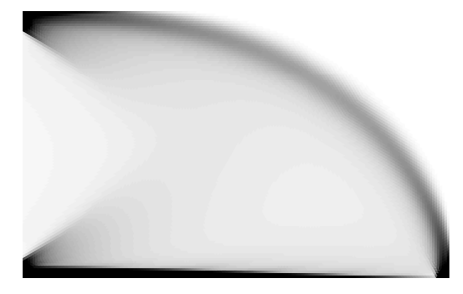

(d) 32,494 elements, $\boldsymbol{V}^{T} \hat{\boldsymbol{\rho}}=0.1580$

Fig. 7 The optimal design the cantilever beam problem using the standard element is independent of mesh.

\subsection{Cantilever beam with circular hole}

A circular hole is now added to the cantilever beam. The beam still has a length of $l=32$ and a height of $h=20$. The centre of the hole is located at $(l / 3, h / 2)$ and has a radius of $h / 3$, see Fig. 9. Identically to the cantilever beam problem, the load is applied over a length of $s=1$ and the magnitude of the load is $P=100$. A yield strength of $f_{y}^{0}=100$ is applied here as well. 


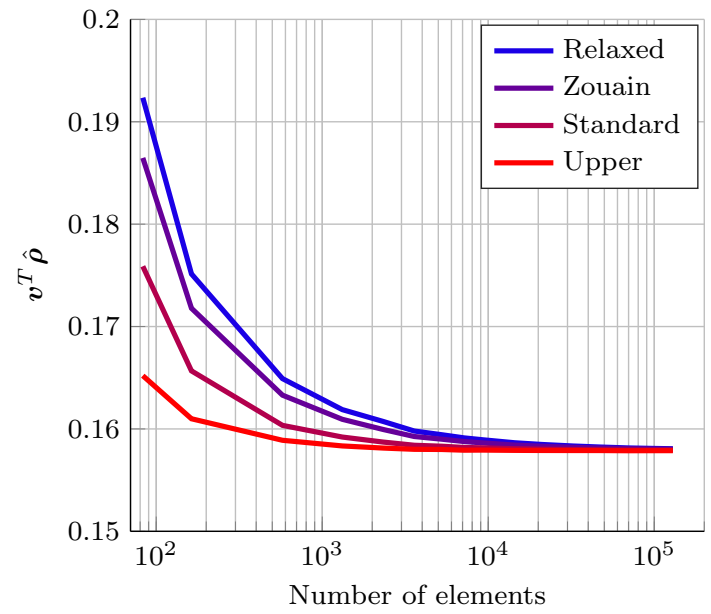

(a)

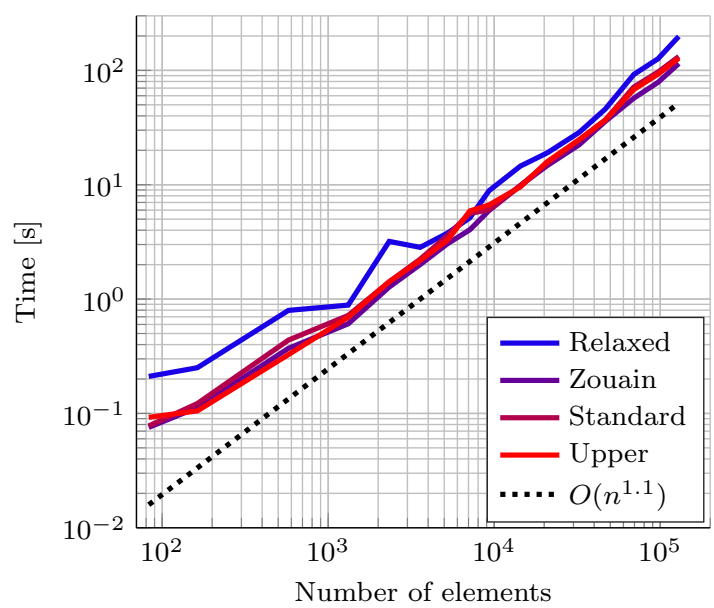

(b)

Fig. 8 Cantilever beam problem: a) Convergence for the four elements, b) computational time for the four elements.

The solution to the dual problem can be interpreted as the displacement of the structure, see Sec. 2.3. Fig. 10 shows the optimal design and scaled displacement field using a rather fine mesh of 100,656 upper bound elements. The addition of the hole leads to a truss-like structure near the left hand side. Near the hole, a significant portion of the available area is utilised. Fig. 10(b) shows the determined displacement field associated with the optimal design. It is observed that both bending and shear contributes considerably to the displacements.

Fig. 11(a) shows that coarse meshes lead to very different volume fractions for the different elements, however, as the mesh is refined a common 


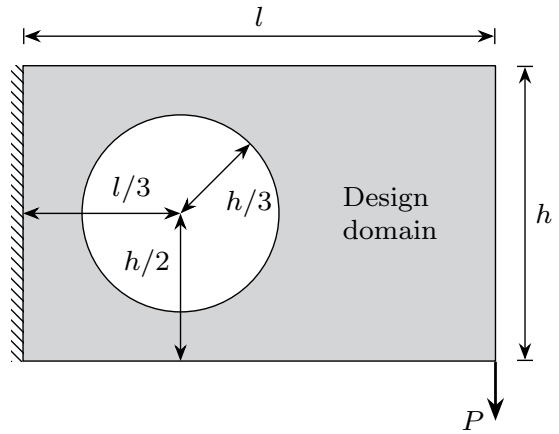

Fig. 9 Cantilever beam with circular hole subjected to a point load at the bottom right corner. Left boundary is assumed fixed.

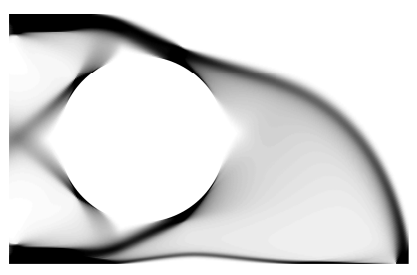

(a) Design

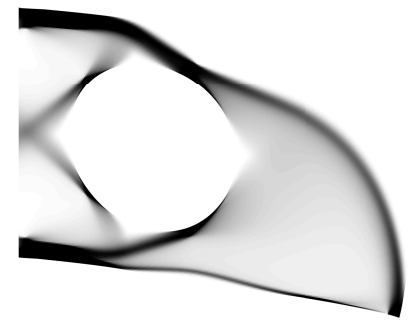

(b) Scaled displacement field

Fig. 10 Optimal design and failure mode of the cantilever beam with circular hole problem using the upper bound element and 100,656 elements: $\boldsymbol{V}^{T} \hat{\boldsymbol{\rho}}=0.2159$.

volume fraction and design are obtained. The time-complexity of the problem is identical to the previous problems as Fig. 11(b) shows.

\subsection{Portal structure}

The fourth and finale example is a portal structure subjected to a point load in the centre. The symmetry of the structure is exploited and only half is modelled. The portal is supported at the bottom of the domain as shown in Fig. 12.

The portal structure has a length of $l=16$ and a height of $h=8$. The width of the beam and column parts of the domain is given by $b=2$. The point load $P=25$ is distributed a length of $s=1$ in the centre, and $f_{y}^{0}=100$ is used here as well.

Fig. 13(a) shows the optimal design of the rather narrow portal structure. The design features wide flanges near midspan. It is seen that between the corner and midspan, a hinge is present since the moment is close to zero and 


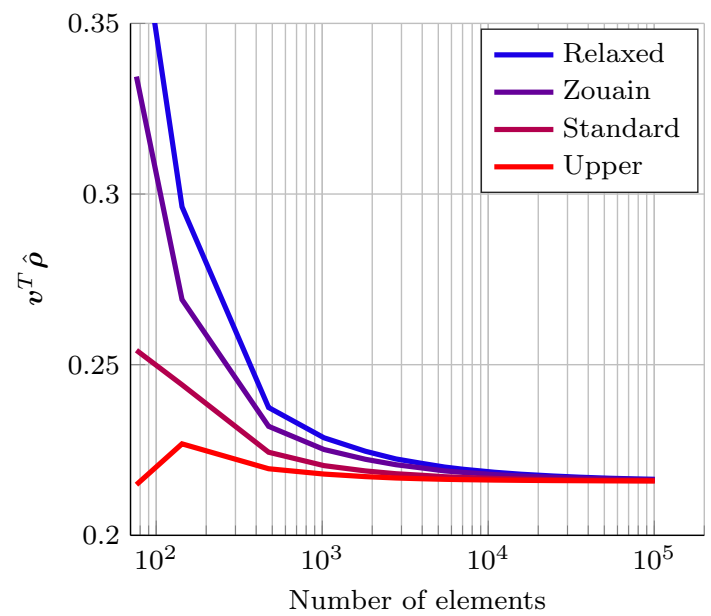

(a)

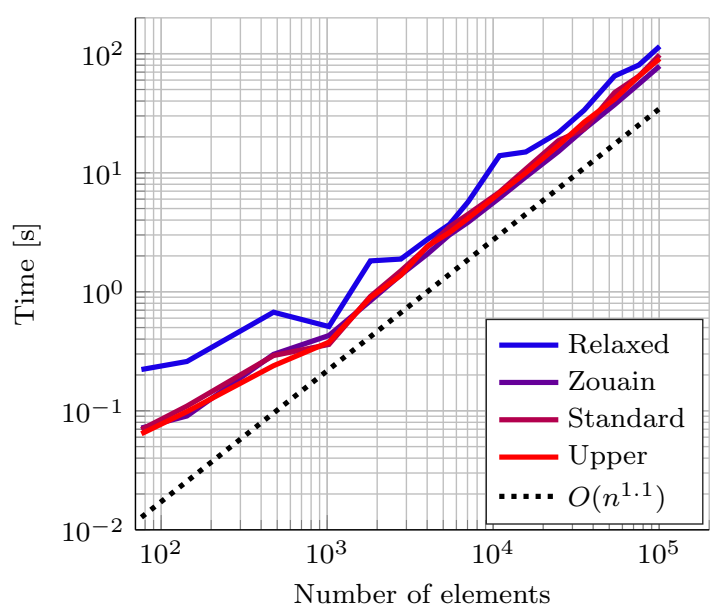

(b)

Fig. 11 Cantilever beam problem with circular hole: a) Convergence for the four elements, b) computational time for the four elements.

only transfer of shear is needed. A moment-resisting corner is established by use of a curved flange and a grey-scale web to carry shear and axial forces.

For comparison, the supports at the bottom are also modelled as rollers, i.e. only supported in the $y$-direction, and the optimal design is shown in Fig. 13(b). Compared to the fixed supports, the problem with roller supports require considerably more material to carry the same load. The column part of the domain will be subjected to a constant moment, which the design also reflects as seen in Fig. 13(b). Moreover, it is observed that the hinge is located closer to the column for this design, thus, the moment is larger at midspan. 


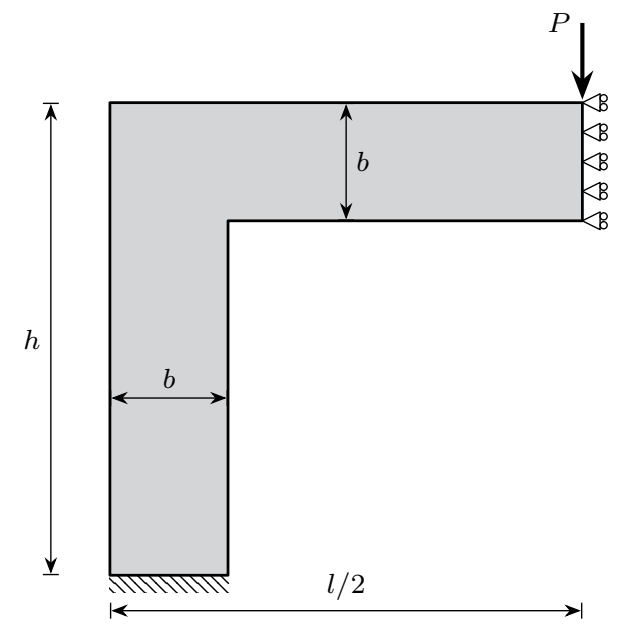

Fig. 12 Portal structure subjected to a point load in the centre. The symmetry is exploited and only half of the structure is modelled.

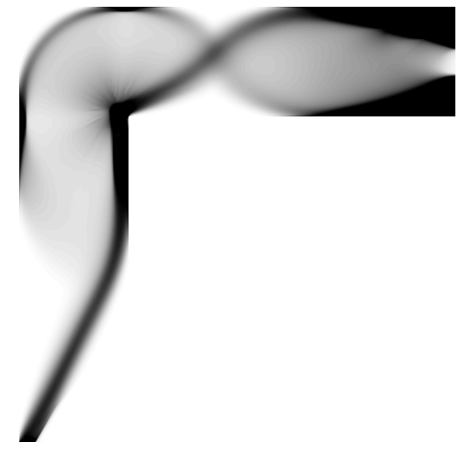

(a) Fixed supports, $\boldsymbol{V}^{T} \hat{\boldsymbol{\rho}}=0.3306$.

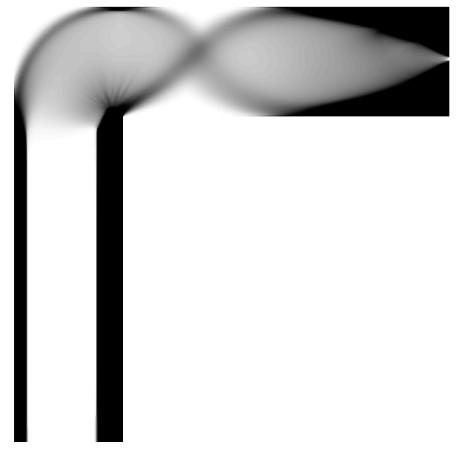

(b) Roller supports, $\boldsymbol{V}^{T} \hat{\boldsymbol{\rho}}=0.4140$.

Fig. 13 Optimal design of the portal structure problem for different support conditions using the relaxed lower bound element and 233,100 elements.

Fig. 14(a) shows that all four elements approach the same design as the mesh is refined. Moreover, it is observed that the design of the upper bound element approach the optimal design from below for all meshes, while the remaining three elements approach from above. Fig. 14(b) displays that the portal structure problem exhibits a polynomial time-complexity identically to the other examples. 


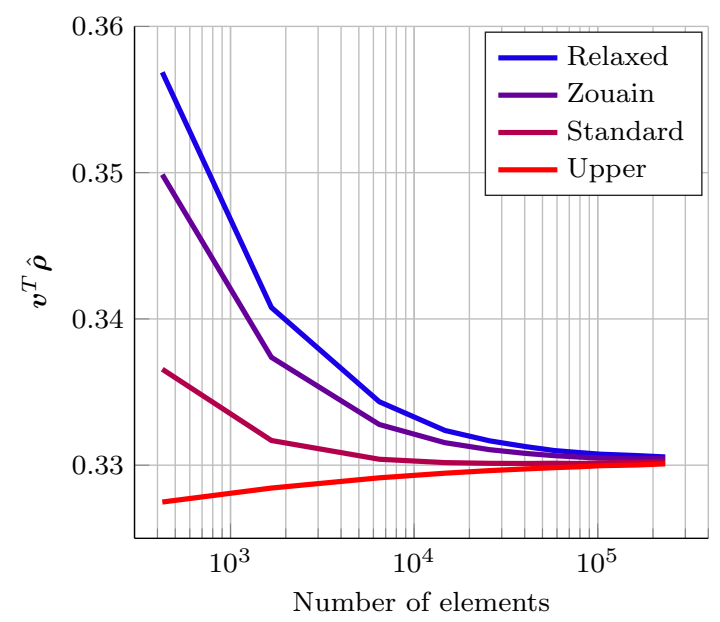

(a)

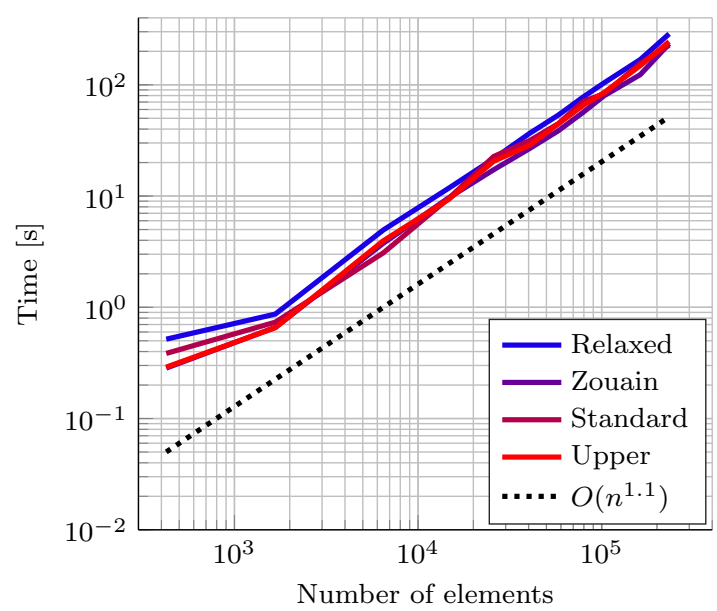

(b)

Fig. 14 Portal structure problem: a) Convergence for the four elements, b) computational time for the four elements.

\section{Conclusions}

A strength-based formulation for topology optimisation of structures for von Mises materials has been presented. The formulation is based on finite element limit analysis and the governing equations can be derived from the work equation and are based on strength rather than stiffness. The stress constraints are enforced locally for every stress interpolation point.

The resulting mathematical problem is a convex program, which can be solved remarkably efficient using interior point methods. This was illustrated with the four examples where optimisation problems with hundred thousand 
elements and millions of variables were solved in a few minutes on a laptop computer. It was shown that the polynomial time-complexity of the interior point methods is rather close to being linear for the presented formulation. Moreover, the fact that the formulation is a convex program ensures that the solution is indeed the global solution.

The examples also displayed that the optimal design is mesh-independent. Refining the mesh will give a more refined design, but the overall design is unchanged. All four plane stress elements converge rather quickly towards a common design, with the upper bound and standard elements being the most accurate. Moreover, the use of upper bound and relaxed lower bound elements makes it possible to bound the optimal design.

Finally, while the present paper only is concerned with the von Mises criterion, any convex yield function will fit the presented framework. This even includes non-smooth yield functions such as Tresca and Mohr-Coulomb criteria which can be formulated exact in two dimensions using second-order constraints.

The convex nature of the formulation means that penalisation such as the SIMP approach cannot be incorporated. Therefore, grey-scale designs are currently unavoidable using the present framework. Using a discrete integer formulation would guarantee black-white designs, however, as discussed the NP-hardness of integer optimisation makes this undesirable for large-scale problems. Alternatively, the problem can be extended to non-convex programming, incorporating penalisation or similar. The convex solution can be used as starting guess and the degree of penalisation can be increased over several iterations.

As a final note it should be mentioned that the framework of finite element limit analysis is widely used for three-dimensional problems in geotechnical engineering. A generalisation to three-dimensional problems should therefore be straightforward as finite elements and yield functions already have been developed for finite element limit analysis. Such generalisation will be considered for future publications.

\section{Acknowledgement}

The authors would like to thank the ALECTIA Foundation and Innovation Fund Denmark for financial support of the research presented in this paper.

\section{References}

1. Anderheggen, E., Knöpfel, H.: Finite element limit analysis using linear programming. International Journal of Solids and Structures 8, 1413-1431 (1972)

2. Andersen, E.D., Roos, C., Terlaky, T.: On implementing a primal-dual interior-point method for conic quadratic optimization. Mathematical Programming 95(2), 249-277 (2003)

3. Bendsøe, M.P.: Optimization of structural topology, shape, and material, vol. 414 Springer (1995) 
4. Bendsøe, M.P., Kikuchi, N.: Generating optimal topologies in structural design using a homogenization method. Computer methods in applied mechanics and engineering 71(2), 197-224 (1988)

5. Bendsøe, M.P., Sigmund, O.: Topology Optimization: Theory, Methods and Applications. Springer (2003)

6. Bisbos, C., Pardalos, P.: Second-order cone and semidefinite representations of material failure criteria. Journal of Optimization Theory and Applications 134(2), 275-301 (2007)

7. Borges, L.A., Zouain, N., Huespe, A.E.: A nonlinear optimization procedure for limit analysis. European Journal of Mechanics Series A Solids 15, 487-512 (1996)

8. Boyd, S.P., Vandenberghe, L.: Convex Optimization. Cambridge University Press (2004)

9. Bruggi, M., Duysinx, P.: A stress-based approach to the optimal design of structures with unilateral behavior of material or supports. Structural and Multidisciplinary Optimization 48(2), 311-326 (2013)

10. Deaton, J.D., Grandhi, R.V.: A survey of structural and multidisciplinary continuum topology optimization: post 2000. Structural and Multidisciplinary Optimization 49(1), $1-38(2014)$

11. Dorn, W.S., Gomory, R.E., Greenberg, H.J.: Automatic design of optimal structures. Journal de Mecanique 3, 25-52 (1964)

12. Duysinx, P., Sigmund, O.: New developments in handling stress constraints in optimal material distribution. In: Proc of the 7th AIAA/USAF/NASAISSMO Symp on Multidisciplinary Analysis and Optimization, vol. 1, pp. 1501-1509 (1998)

13. Duysinx, P., Van Miegroet, L., Lemaire, E., Brüls, O., Bruyneel, M.: Topology and generalized shape optimization: Why stress constraints are so important? International Journal for Simulation and Multidisciplinary Design Optimization 2(4), 253-258 (2008)

14. Engwirda, D.: Locally optimal delaunay-refinement and optimisation-based mesh generation. Ph.D. thesis, University of Sydney (2014)

15. Haftka, R.T., Grandhi, R.V.: Structural shape optimization - a survey. Computer methods in applied mechanics and engineering 57(1), 91-106 (1986)

16. Hegemier, G., Prager, W.: On Michell trusses. International Journal of Mechanical Sciences 11(2), 209-215 (1969)

17. Herfelt, M.A., Poulsen, P.N., Hoang, L.C., Jensen, J.F.: Lower bound equilibrium element and submodel for shear joints in precast concrete structures. Engineering Structures 135, $1-9$ (2017)

18. Kammoun, Z., Smaoui, H.: A direct approach for continuous topology optimization subject to admissible loading. Comptes Rendus Mécanique 342(9), 520-531 (2014)

19. Karmarkar, N.: A new polynomial-time algorithm for linear programming. Combinatorica 4, 373-395 (1984)

20. Kohn, R.V., Strang, G.: Optimal design and relaxation of variational problems, i. Communications on pure and applied mathematics 39(1), 113-137 (1986)

21. Krabbenhøft, K.: Shell finite element (2016). Optum Computational Engineering

22. Krabbenhøft, K., Lyamin, A., Sloan, S.: Formulation and solution of some plasticity problems as conic programs. International Journal of Solids and Structures 44(5), 1533-1549 (2007)

23. Lobo, M.S., Vandenberghe, L., Boyd, S., Lebret, H.: Applications of second-order cone programming. Linear algebra and its applications 284(1), 193-228 (1998)

24. Lyamin, A.V., Sloan, S.W.: Upper bound limit analysis using linear finite elements and non-linear programming. International Journal for Numerical and Analytical Methods in Geomechanics 26(2), 181-216 (2002)

25. Makrodimopoulos, A., Martin, C.: Lower bound limit analysis of cohesive-frictional materials using second-order cone programming. International Journal for Numerical Methods in Engineering 66(4), 604-634 (2006)

26. Makrodimopoulos, A., Martin, C.: Upper bound limit analysis using simplex strain elements and second-order cone programming. International Journal for Numerical and Analytical Methods in Geomechanics (2007)

27. Michell, A.G.M.: Lviii. the limits of economy of material in frame-structures. The London, Edinburgh, and Dublin Philosophical Magazine and Journal of Science 8(47), 589-597 (1904) 
28. MOSEK ApS: MOSEK MATLAB toolbox. Release 8.0.0.81 (2017). Available at https : //www.mosek.com/

29. Nemhauser, G.L., Wolsey, L.A.: Integer programming and combinatorial optimization. Wiley, Chichester. GL Nemhauser, MWP Savelsbergh, GS Sigismondi (1992). Constraint Classification for Mixed Integer Programming Formulations. COAL Bulletin 20 , 8-12 (1988)

30. Nesterov, Y., Nemirovsky, A.: A general approach to polynomial-time algorithms design for convex programming (1988). Report, Central Economical and Mathematical Institute, USSR Academy of Sciences, Moscow

31. Olhoff, N., Taylor, J.: On structural optimization. Journal of Applied Mechanics 50(4b), 1139-1151 (1983)

32. Pedersen, P.: Some general optimal design results using anisotropic, power law nonlinear elasticity. Structural optimization 15(2), 73-80 (1998)

33. Poulsen, P.N., Damkilde, L.: Limit state analysis of reinforced concrete plates subjected to in-plane forces. International Journal of Solids and Structures 37, 6011-6029 (2000)

34. Prager, W., Rozvany, G.: Optimal layout of grillages. Journal of Structural Mechanics 5(1), 1-18 (1977)

35. Prager, W., Rozvany, G.I.: Optimization of structural geometry. In: Dynamical systems, pp. 265-293. Elsevier (1977)

36. Prager, W., Shield, R.: A general theory of optimal plastic design. Journal of Applied Mechanics 34(1), 184-186 (1967)

37. Prager, W., Taylor, J.E.: Problems of optimal structural design. Journal of Applied Mechanics 35(1), 102-106 (1968)

38. Rozvany, G.I., Prager, W.: A new class of structural optimization problems: optimal archgrids. Computer methods in applied mechanics and engineering 19(1), 127-150 (1979)

39. Rozvany, G.I., Zhou, M., Birker, T.: Generalized shape optimization without homogenization. Structural optimization 4(3-4), 250-252 (1992)

40. Sigmund, O.: A 99 line topology optimization code written in matlab. Structural and multidisciplinary optimization 21(2), 120-127 (2001)

41. Sigmund, O., Petersson, J.: Numerical instabilities in topology optimization: a survey on procedures dealing with checkerboards, mesh-dependencies and local minima. Structural optimization 16(1), 68-75 (1998)

42. Sloan, S.W.: Lower bound limit analysis using finite elements and linear programming. International Journal for Numerical and Analytical Methods in Geomechanics 12, 61-77 (1988)

43. Sloan, S.W.: Upper bound limit analysis using finite elements and linear programming. International Journal for Numerical and Analytical Methods in Geomechanics 13, 263$282(1989)$

44. Svanberg, K.: The method of moving asymptotesa new method for structural optimization. International journal for numerical methods in engineering 24(2), 359-373 (1987)

45. Terlaky, T.: Interior point methods of mathematical programming, vol. 5. Springer Science \& Business Media (2013)

\section{A Equilibrium matrices for the plane stress elements}

A.1 The Zouain element

$$
\hat{\mathbf{B}}^{T}=-\frac{1}{6}\left[\begin{array}{ccc}
\tilde{\mathbf{P}}_{1} & 0 & 0 \\
0 & \tilde{\mathbf{P}}_{2} & 0 \\
0 & 0 & \tilde{\mathbf{P}}_{3} \\
-\tilde{\mathbf{P}}_{1} & \tilde{\mathbf{P}}_{3}-\tilde{\mathbf{P}}_{1} & \tilde{\mathbf{P}}_{2}-\tilde{\mathbf{P}}_{1} \\
\tilde{\mathbf{P}}_{3}-\tilde{\mathbf{P}}_{2} & -\tilde{\mathbf{P}}_{2} & \tilde{\mathbf{P}}_{1}-\tilde{\mathbf{P}}_{2} \\
\tilde{\mathbf{P}}_{2}-\tilde{\mathbf{P}}_{3} & \tilde{\mathbf{P}}_{1}-\tilde{\mathbf{P}}_{3} & -\tilde{\mathbf{P}}_{3}
\end{array}\right]
$$


A.2 Standard six-node element

$$
\hat{\mathbf{B}}^{T}=-\frac{1}{18}\left[\begin{array}{ccc}
5 \tilde{\mathbf{P}}_{1} & -\tilde{\mathbf{P}}_{1} & -\tilde{\mathbf{P}}_{1} \\
-\tilde{\mathbf{P}}_{2} & 5 \tilde{\mathbf{P}}_{2} & -\tilde{\mathbf{P}}_{2} \\
-\tilde{\mathbf{P}}_{3} & -\tilde{\mathbf{P}}_{3} & 5 \tilde{\mathbf{P}}_{3} \\
-2 \tilde{\mathbf{P}}_{1} & 2 \tilde{\mathbf{P}}_{2}+8 \tilde{\mathbf{P}}_{3} & 2 \tilde{\mathbf{P}}_{3}+8 \tilde{\mathbf{P}}_{2} \\
2 \tilde{\mathbf{P}}_{1}+8 \tilde{\mathbf{P}}_{3} & -2 \tilde{\mathbf{P}}_{2} & 2 \tilde{\mathbf{P}}_{3}+8 \tilde{\mathbf{P}}_{1} \\
2 \tilde{\mathbf{P}}_{1}+8 \tilde{\mathbf{P}}_{2} & 2 \tilde{\mathbf{P}}_{2}+8 \tilde{\mathbf{P}}_{1} & -2 \tilde{\mathbf{P}}_{3}
\end{array}\right]
$$

A.3 Upper bound element

$$
\hat{\mathbf{B}}^{T}=-\frac{1}{6}\left[\begin{array}{ccc}
3 \tilde{\mathbf{P}}_{1} & -\tilde{\mathbf{P}}_{1} & -\tilde{\mathbf{P}}_{1} \\
-\tilde{\mathbf{P}}_{2} & 3 \tilde{\mathbf{P}}_{2} & -\tilde{\mathbf{P}}_{2} \\
-\tilde{\mathbf{P}}_{3} & -\tilde{\mathbf{P}}_{3} & 3 \tilde{\mathbf{P}}_{3} \\
0 & 4 \tilde{\mathbf{P}}_{3} & 4 \tilde{\mathbf{P}}_{2} \\
4 \tilde{\mathbf{P}}_{3} & 0 & 4 \tilde{\mathbf{P}}_{1} \\
4 \tilde{\mathbf{P}}_{2} & 4 \tilde{\mathbf{P}}_{1} & 0
\end{array}\right]
$$

\section{B Tabulated results}

Table 1 Minimum volume and computational time for the MBB-beam problem.

\begin{tabular}{rcccccccc}
\hline & \multicolumn{2}{c}{ Relaxed lower } & \multicolumn{2}{c}{ Zouain } & \multicolumn{2}{c}{ Standard } & \multicolumn{2}{c}{ Upper } \\
$n e l$ & $\boldsymbol{v}^{T} \boldsymbol{\rho}$ & Time $[\mathrm{s}]$ & $\boldsymbol{v}^{T} \boldsymbol{\rho}$ & Time $[\mathrm{s}]$ & $\boldsymbol{v}^{T} \boldsymbol{\rho}$ & Time $[\mathrm{s}]$ & $\boldsymbol{v}^{T} \boldsymbol{\rho}$ & Time $[\mathrm{s}]$ \\
\hline 136 & 0.2336 & 0.26 & 0.2279 & 0.09 & 0.2168 & 0.11 & 0.2103 & 0.11 \\
152 & 0.2267 & 0.27 & 0.2216 & 0.09 & 0.2126 & 0.11 & 0.2073 & 0.10 \\
352 & 0.2079 & 0.42 & 0.2053 & 0.20 & 0.2012 & 0.25 & 0.1991 & 0.19 \\
737 & 0.2025 & 1.03 & 0.2007 & 0.52 & 0.1984 & 0.62 & 0.1971 & 0.43 \\
1,251 & 0.2004 & 0.75 & 0.1991 & 0.97 & 0.1974 & 0.57 & 0.1965 & 0.46 \\
1,936 & 0.1992 & 1.33 & 0.1981 & 0.88 & 0.1968 & 1.15 & 0.1961 & 1.06 \\
2,775 & 0.1985 & 1.88 & 0.1976 & 1.31 & 0.1966 & 1.67 & 0.1960 & 1.67 \\
3,782 & 0.1979 & 2.60 & 0.1972 & 1.83 & 0.1964 & 2.14 & 0.1959 & 2.22 \\
4,988 & 0.1975 & 3.32 & 0.1969 & 2.58 & 0.1962 & 3.32 & 0.1959 & 2.89 \\
7,767 & 0.1970 & 5.38 & 0.1966 & 4.78 & 0.1961 & 4.67 & 0.1958 & 5.35 \\
11,073 & 0.1967 & 8.29 & 0.1964 & 7.00 & 0.1960 & 7.95 & 0.1958 & 7.42 \\
17,398 & 0.1964 & 14.10 & 0.1962 & 13.00 & 0.1959 & 12.90 & 0.1958 & 11.06 \\
24,925 & 0.1963 & 21.50 & 0.1961 & 13.89 & 0.1959 & 16.99 & 0.1958 & 16.37 \\
37,354 & 0.1961 & 33.43 & 0.1960 & 23.09 & 0.1958 & 27.45 & 0.1957 & 28.20 \\
51,903 & 0.1960 & 50.86 & 0.1959 & 32.86 & 0.1958 & 39.94 & 0.1957 & 38.76 \\
68,889 & 0.1959 & 74.80 & 0.1958 & 54.15 & 0.1958 & 60.12 & 0.1957 & 56.69 \\
\hline
\end{tabular}


Table 2 Minimum volume and computational time for the cantilever problem.

\begin{tabular}{rrrrrrrrr}
\hline & \multicolumn{2}{c}{ Relaxed lower } & \multicolumn{2}{c}{ Zouain } & \multicolumn{2}{c}{ Standard } & \multicolumn{2}{c}{ Upper } \\
$n e l$ & $\boldsymbol{v}^{T} \boldsymbol{\rho}$ & Time [s] & $\boldsymbol{v}^{T} \boldsymbol{\rho}$ & Time $[\mathrm{s}]$ & $\boldsymbol{v}^{T} \boldsymbol{\rho}$ & Time $[\mathrm{s}]$ & $\boldsymbol{v}^{T} \boldsymbol{\rho}$ & Time $[\mathrm{s}]$ \\
\hline 83 & 0.1923 & 0.21 & 0.1865 & 0.08 & 0.1759 & 0.08 & 0.1652 & 0.09 \\
163 & 0.1751 & 0.25 & 0.1718 & 0.11 & 0.1657 & 0.12 & 0.1610 & 0.11 \\
576 & 0.1649 & 0.80 & 0.1633 & 0.37 & 0.1603 & 0.44 & 0.1589 & 0.33 \\
1,320 & 0.1619 & 0.89 & 0.1609 & 0.61 & 0.1592 & 0.72 & 0.1583 & 0.69 \\
2,331 & 0.1607 & 3.19 & 0.1599 & 1.27 & 0.1587 & 1.42 & 0.1581 & 1.42 \\
3,582 & 0.1598 & 2.84 & 0.1593 & 2.01 & 0.1584 & 2.23 & 0.1580 & 2.17 \\
5,238 & 0.1594 & 3.78 & 0.1590 & 3.05 & 0.1583 & 3.60 & 0.1580 & 3.28 \\
7,140 & 0.1591 & 5.14 & 0.1588 & 4.04 & 0.1582 & 5.62 & 0.1579 & 5.91 \\
9,297 & 0.1589 & 8.85 & 0.1586 & 5.94 & 0.1582 & 6.03 & 0.1579 & 6.65 \\
14,384 & 0.1587 & 14.56 & 0.1584 & 9.83 & 0.1581 & 9.85 & 0.1579 & 9.58 \\
20,826 & 0.1585 & 18.97 & 0.1583 & 14.68 & 0.1580 & 15.30 & 0.1579 & 15.89 \\
32,494 & 0.1583 & 28.67 & 0.1582 & 22.37 & 0.1580 & 24.56 & 0.1579 & 24.81 \\
46,654 & 0.1582 & 45.86 & 0.1581 & 35.79 & 0.1580 & 36.73 & 0.1579 & 36.74 \\
69,365 & 0.1581 & 92.21 & 0.1581 & 57.14 & 0.1579 & 71.59 & 0.1579 & 68.09 \\
97,056 & 0.1581 & 126.44 & 0.1580 & 78.68 & 0.1579 & 96.75 & 0.1579 & 92.67 \\
129,387 & 0.1581 & 198.35 & 0.1580 & 114.76 & 0.1579 & 132.25 & 0.1579 & 127.73 \\
\hline
\end{tabular}

Table 3 Minimum volume and computational time for the cantilever beam with circular hole problem.

\begin{tabular}{rcccccccc}
\hline & \multicolumn{2}{c}{ Relaxed lower } & \multicolumn{2}{c}{ Zouain } & \multicolumn{2}{c}{ Standard } & \multicolumn{2}{c}{ Upper } \\
$n e l$ & $\boldsymbol{v}^{T} \boldsymbol{\rho}$ & Time $[\mathrm{s}]$ & $\boldsymbol{v}^{T} \boldsymbol{\rho}$ & Time $[\mathrm{s}]$ & $\boldsymbol{v}^{T} \boldsymbol{\rho}$ & Time $[\mathrm{s}]$ & $\boldsymbol{v}^{T} \boldsymbol{\rho}$ & Time $[\mathrm{s}]$ \\
\hline 76 & 0.3862 & 0.22 & 0.3344 & 0.07 & 0.2542 & 0.07 & 0.2148 & 0.06 \\
143 & 0.2963 & 0.26 & 0.2691 & 0.09 & 0.2441 & 0.11 & 0.2268 & 0.10 \\
475 & 0.2375 & 0.67 & 0.2319 & 0.30 & 0.2244 & 0.29 & 0.2195 & 0.24 \\
1,024 & 0.2286 & 0.51 & 0.2252 & 0.43 & 0.2204 & 0.36 & 0.2180 & 0.38 \\
1,820 & 0.2246 & 1.82 & 0.2223 & 0.84 & 0.2189 & 0.91 & 0.2172 & 0.89 \\
2,776 & 0.2224 & 1.88 & 0.2207 & 1.39 & 0.2181 & 1.50 & 0.2168 & 1.38 \\
4,011 & 0.2211 & 2.75 & 0.2197 & 2.07 & 0.2176 & 2.37 & 0.2166 & 2.41 \\
5,444 & 0.2200 & 3.67 & 0.2189 & 3.00 & 0.2172 & 3.50 & 0.2164 & 3.14 \\
7,060 & 0.2194 & 5.69 & 0.2184 & 3.84 & 0.2170 & 4.48 & 0.2163 & 4.14 \\
10,903 & 0.2185 & 13.90 & 0.2178 & 6.13 & 0.2167 & 6.86 & 0.2162 & 6.74 \\
15,714 & 0.2179 & 14.94 & 0.2174 & 9.22 & 0.2165 & 10.74 & 0.2161 & 9.97 \\
24,693 & 0.2174 & 21.68 & 0.2170 & 15.06 & 0.2163 & 18.58 & 0.2160 & 16.97 \\
35,292 & 0.2170 & 33.50 & 0.2167 & 23.19 & 0.2162 & 23.29 & 0.2160 & 26.56 \\
53,840 & 0.2167 & 64.95 & 0.2165 & 37.32 & 0.2161 & 47.10 & 0.2160 & 41.56 \\
75,432 & 0.2165 & 80.29 & 0.2164 & 55.72 & 0.2161 & 64.74 & 0.2159 & 64.75 \\
100,656 & 0.2164 & 115.20 & 0.2163 & 78.87 & 0.2160 & 97.83 & 0.2159 & 90.87 \\
\hline
\end{tabular}


Table 4 Minimum volume and computational time for the portal structure problem with fixed supports.

\begin{tabular}{|c|c|c|c|c|c|c|c|c|}
\hline \multirow[b]{2}{*}{ nel } & \multicolumn{2}{|c|}{ Relaxed lower } & \multicolumn{2}{|c|}{ Zouain } & \multicolumn{2}{|c|}{ Standard } & \multicolumn{2}{|c|}{ Upper } \\
\hline & $\boldsymbol{v}^{T} \boldsymbol{\rho}$ & Time $[\mathrm{s}]$ & $\boldsymbol{v}^{T} \boldsymbol{\rho}$ & Time $[\mathrm{s}]$ & $\boldsymbol{v}^{T} \boldsymbol{\rho}$ & Time $[\mathrm{s}]$ & $\boldsymbol{v}^{T} \boldsymbol{\rho}$ & Time $[\mathrm{s}]$ \\
\hline 425 & 0.3569 & 0.52 & 0.3499 & 0.28 & 0.3366 & 0.38 & 0.3275 & 0.29 \\
\hline 1,670 & 0.3408 & 0.87 & 0.3374 & 0.66 & 0.3317 & 0.74 & 0.3284 & 0.66 \\
\hline 6,466 & 0.3343 & 4.95 & 0.3328 & 3.76 & 0.3304 & 3.08 & 0.3291 & 3.96 \\
\hline 14,716 & 0.3324 & 11.79 & 0.3315 & 9.66 & 0.3302 & 9.55 & 0.3295 & 9.35 \\
\hline 25,798 & 0.3317 & 21.62 & 0.3311 & 17.23 & 0.3301 & 22.67 & 0.3296 & 20.56 \\
\hline 40,548 & 0.3313 & 36.66 & 0.3308 & 26.76 & 0.3301 & 31.57 & 0.3297 & 28.66 \\
\hline 58,038 & 0.3310 & 53.41 & 0.3307 & 38.60 & 0.3301 & 44.73 & 0.3298 & 44.55 \\
\hline 79,430 & 0.3309 & 77.82 & 0.3306 & 56.68 & 0.3301 & 71.17 & 0.3299 & 65.54 \\
\hline 103,160 & 0.3308 & 104.19 & 0.3305 & 79.78 & 0.3302 & 82.85 & 0.3300 & 85.27 \\
\hline 162,178 & 0.3307 & 168.58 & 0.3304 & 123.28 & 0.3302 & 156.06 & 0.3300 & 148.61 \\
\hline 233,100 & 0.3306 & 286.91 & 0.3305 & 233.59 & 0.3302 & 226.84 & 0.3301 & 244.36 \\
\hline
\end{tabular}

